\title{
Transversity in hard exclusive electroproduction of pions
}

\section{Peter Kroll ${ }^{* \dagger}$}

Universitaet Wuppertal

E-mail: kroll@physik.uni-wuppertal.de

In this talk it is reported on an analysis of hard exclusive $\pi^{+}$electroproduction within the handbag approach. Particular emphasis is laid on single-spin asymmetries. It is argued that a recent HERMES measurement of asymmetries measured with a transversely polarized target clearly indicate the occurence of strong contributions from transversely polarized photons. Within the handbag approach such $\gamma_{T}^{*} \rightarrow \pi$ transitions are described by the transversity GPDs accompanied by a twist3 pion wave function. It is shown that this handbag approach leads to results on cross sections and single-spin asymmetries in fair agreement with experiment.

XVIII International Workshop on Deep-Inelastic Scattering and Related Subjects, DIS 2010 April 19-23, 2010

Firenze, Italy

\footnotetext{
${ }^{*}$ Speaker.

${ }^{\dagger}$ This work is supported in part by the BMBF under contract 06RY258.
} 
In this article it will be reported upon an analysis of hard exclusive electroproduction of positively charged pions [1] within the frame work of the so-called handbag approach which offers a partonic description of meson electroproduction provided the virtuality of the exchanged photon, $Q^{2}$, is sufficiently large. The theoretical basis of the handbag approach is the factorization of the process amplitudes in a hard partonic subprocess and soft hadronic matrix elements, the so-called generalized parton distributions (GPDs), as well as wave functions for the produced mesons, see Fig. 1. In collinear approximation factorization has been shown $[2,3]$ to hold rigorously for exclusive meson electroproduction in the limit $Q^{2} \rightarrow \infty$. It has also been shown that the transitions from a longitudinally polarized photon to the pion, $\gamma_{L}^{*} \rightarrow \pi$, dominates at large $Q^{2}$. Transitions from transversely polarized photons to the pion are suppressed by inverse powers of the hard scale. In Ref. [1] a variant of the handbag approach is utilized for the interpretation of the data in which the subprocess amplitudes are calculated within the modified perturbative approach [4]. In this approach the quark transverse momenta are retained in the subprocess and Sudakov suppressions are taken into account. The partons are still emitted and re-absorbed by the nucleon collinearly. It has been shown [5] that within this handbag approach the data on cross sections and spin density matrix elements for vector-meson production are well fitted for small values of skewness ( $\xi \simeq x_{B j} / 2 \lesssim 0.1$ ).

In $\pi^{+}$electroproduction there is also a contribution from pion exchange. Indeed this is the process in which the electromagnetic form factor of the pion can be measured. The pion pole contributes to the GPD as

$$
\widetilde{E}_{\text {pole }}^{u}=-\widetilde{E}_{\text {pole }}^{d}=\Theta(|\bar{x}| \leq \xi) \frac{F_{P}(t)}{4 \xi} \Phi_{\pi}((\bar{x}+\xi) /(2 \xi)),
$$

where $F_{P}$ is the pseudoscalar form factor and $\Phi_{\pi}$ the pion distribution amplitude. Working out the graph shown in Fig. 1 from the GPD (1) one obtains the pion-pole term comprising only the perturbative part of the pion form factor which underestimates its experimental value [6] by about a factor of three for $Q^{2}$ in the range of $2-5 \mathrm{GeV}^{2}$. Obviously, the use of the perturbative result only
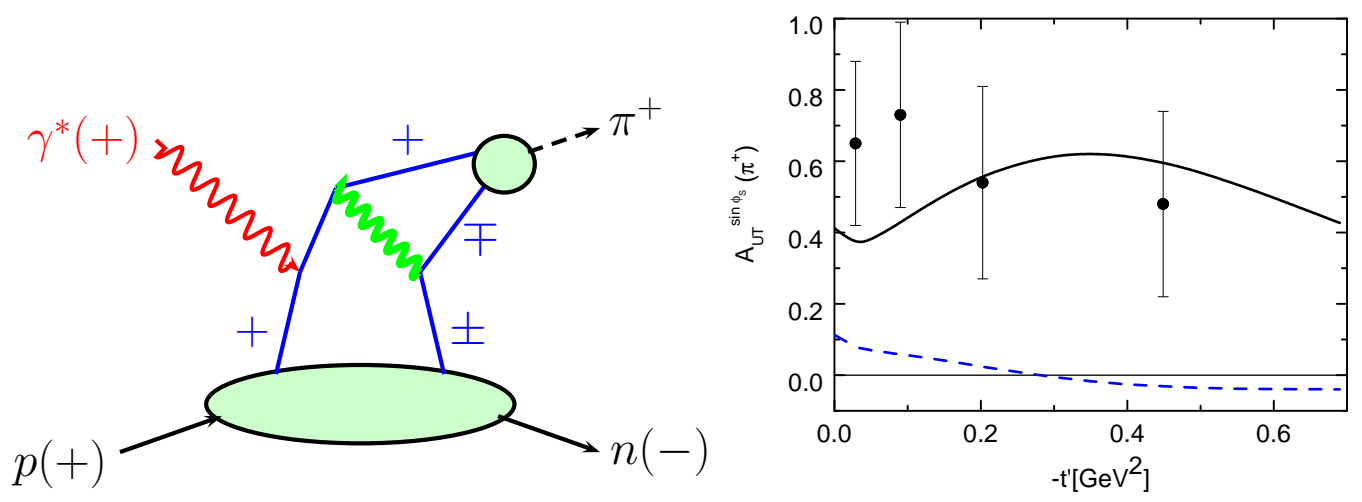

Figure 1: A typical lowest order Feynman graph for pion electroproduction. The signs indicate helicity labels for the contribution from transversity GPDs to the amplitude $\mathscr{M}_{0-,++}$, see text.

Figure 2: The $\sin \phi_{s}$ moment for a transversely polarized target at $Q^{2} \simeq 2.45 \mathrm{GeV}^{2}$ and $W=3.99 \mathrm{GeV}$ for $\pi^{+}$production. The predictions from the handbag approach [1] are shown as a solid line. The dashed line is obtained disregarding the twist-3 contribution. Data are taken from Ref. [7]. 
is in conflict with the very idea of measuring the pion form factor. In [1] therefore the contribution from the pion pole is calculated directly as pion exchange between the nucleon vertex and the photon-pion one, employing thereby the experimental value for the pion form factor. It should be noted that also in [8] the full pion form factor is taken into account. This is achieved within the leading-twist, LO perturbative QCD formalism by using an effective coupling constant $\alpha_{s}$ of about 0.8 .

The electroproduction cross sections measured with a transversely or longitudinally polarized target consist of many terms, each can be projected out by $\sin \varphi$ moments where $\varphi$ is a specific linear combination of $\phi$, the azimuthal angle between the lepton and the hadron plane and $\phi_{s}$, the orientation of the target spin vector. A number of these moments have been measured recently [7, 9]. A particularly striking result is the $\sin \phi_{S}$ moment. The data on it, displayed in Fig. 2, exhibit a mild $t$-dependence and do not show any indication for a turnover towards zero for $t^{\prime} \rightarrow 0$. This behavior of $A_{U T}^{\sin \phi_{s}}$ at small $-t^{\prime}$ can only be produced by the interference term $\operatorname{Im}\left[\mathscr{M}_{0-,++}^{*} \mathscr{M}_{0+, 0+}\right]$. Both the contributing amplitudes, one for a transversally and one for a longitudinally polarized photon photon, are helicity non-flip ones and are therefore not forced to vanish in the forward direction by angular momentum conservation. The amplitude $\mathscr{M}_{0-,++}$ has to be sizeable and we have to conclude that there are strong contributions from $\gamma_{T}^{*} \rightarrow \pi$ transitions.

How can this amplitude be modelled in the frame work of the handbag approach? From Fig. 1 where the helicity configurations for the amplitude $\mathscr{M}_{0-,++}$ are indicated, it is clear that contributions from the usual helicity non-flip GPDs $\widetilde{H}$ and $\widetilde{E}$ to this amplitude do not have the properties required by the data on the $\sin \phi_{s}$ moment. For these GPDs the emitted and reabsorbed partons from the nucleon have the same helicity. Consequently, there are net helicity flips of one unit at both the parton-nucleon vertex and the subprocess. Angular momentum conservation therefore forces both parts to vanish as $\sqrt{-t^{\prime}}$ at least. Thus, a contribution from the ordinary GPDs to $\mathscr{M}_{0-,++}$ vanishes $\propto t^{\prime}$. There is a second set of GPDs, the helicity-flip or transversity ones $H_{T}, E_{T}, \ldots[10,11]$ for which the emitted and reabsorbed partons have opposite helicities. As an inspection of Fig. 1 reveals the parton-nucleon vertex as well as the subprocess amplitude $\mathscr{H}_{0-,++}$ are now of helicity non-flip nature and are therefore not forced to vanish in the forward direction. The prize to pay is that quark and antiquark forming the pion have the same helicity. Therefore, the twist- 3 pion wave function is needed instead of the familiar twist- 2 one. The dynamical mechanism building up the amplitude $\mathscr{M}_{0-,++}$ is so of twist-3 accuracy. It has been first proposed in Ref. [12] for photo- and electroproduction of mesons where $-t$ is considered to be the large scale [13].

In Ref. [1] the twist-3 pion wave function is taken from [14] with the three-particle Fock component neglected. This wave function contains a pseudoscalar and a tensor component. The latter one provides a contribution to $\mathscr{M}_{0-,++}$ which is proportional to $t^{\prime} / Q^{2}$ and is neglected. The contribution from the pseudoscalar component to $\mathscr{M}_{0-,++}$ which is proportional to the parameter $\mu_{\pi}=m_{\pi}^{2} /\left(m_{u}+m_{d}\right) \simeq 2 \mathrm{GeV}$ at the scale of $2 \mathrm{GeV}$ as a consequence of the divergency of the axial-vector current ( $m_{u}$ and $m_{d}$ are current quark masses), has the required properties. Although parametrically suppressed by $\mu_{\pi} / Q$ as compared to the longitudinal amplitudes, it is sizeable for $Q$ of the order of a few $\mathrm{GeV}$.

For $\pi^{+}$electroproduction the GPDs, namely $\widetilde{H}$, the non-pole contribution to $\widetilde{E}$ and the most 

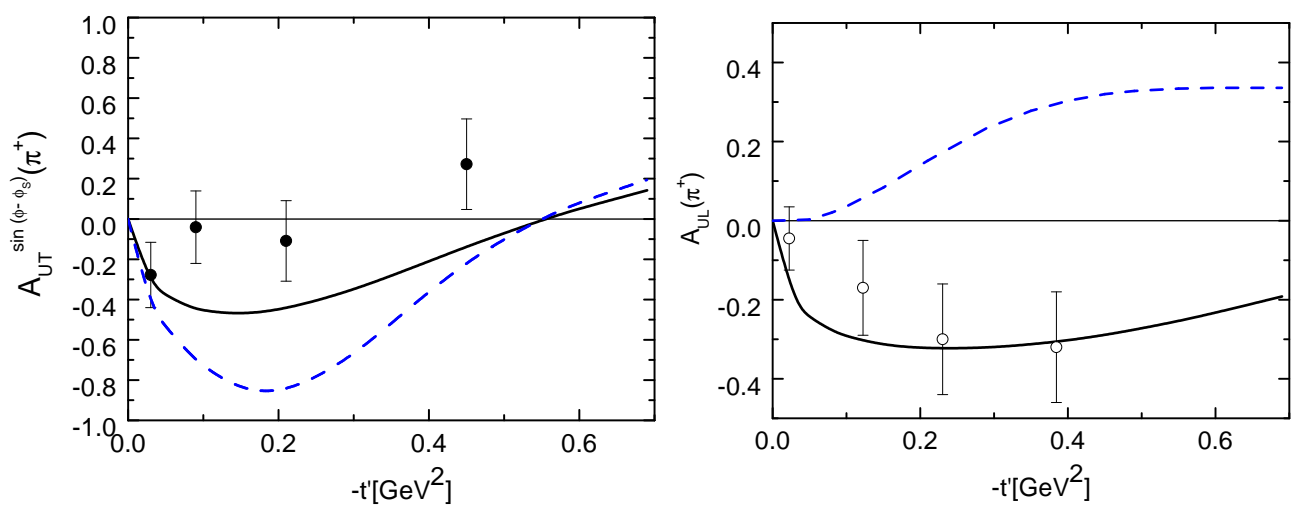

Figure 3: Left: Predictions for the $\sin \left(\phi-\phi_{s}\right)$ moment at $Q^{2}=2.45 \mathrm{GeV}^{2}$ and $W=3.99 \mathrm{GeV}$ shown as solid lines [1]. The dashed line represents the longitudinal contribution to the $\sin \left(\phi-\phi_{s}\right)$ moment. Data are taken from [7].

Figure 4: Right: The asymmetry for a longitudinally polarized target at $Q^{2} \simeq 2.4 \mathrm{GeV}^{2}$ and $W \simeq 4.1 \mathrm{GeV}$. The dashed line is obtained disregarding the twist-3 contribution. Data are taken from [9].

important one of the transversity GPDs, $H_{T}$ contribute in the isovector combination

$$
F^{(3)}=F^{u}-F^{d}
$$

The GPDs are constructed with the help of double distributions ansatz [15] consisting of the product of the zero-skewness GPDs and an appropriate weight functions which generates the skewness dependence. The zero-skewness GPDs are parameterized as their forward limits multiplied by a Regge-like $t$ dependence, $\exp \left[b_{i}-\alpha_{i}^{\prime} \ln x\right] t$. In the case of $\widetilde{H}$ the forward limit is given by the polarized parton distributions while for $H_{T}$ it is the transversity distribution $\delta(x)$ for which the results of an analysis of the asymmetries in semi-inclusive electroproduction have been taken [16]. Finally, the non-pole part of $\widetilde{E}$ is parameterized as

$$
\widetilde{E}_{\text {n.p. }}^{(3)}(x, \xi=t=0)=\widetilde{N}_{\tilde{e}}^{(3)} x^{-0.48}(1-x)^{5},
$$

with the normalization $\widetilde{N}_{\tilde{e}}^{(3)}$ fitted to experiment.

It is shown in [1] that with the described GPDs, the $\pi^{+}$cross sections as measured by HERMES [17] are nicely fitted as well as the transverse target asymmetries [7]. This can be seen for $A_{U T}^{\sin \phi_{s}}$ from Fig. 1. Also the $\sin \left(\phi-\phi_{s}\right)$ moment which is dominantly fed by an interference term of the two amplitudes for longitudinally polarized photons, is fairly well described as is obvious from Fig. 3. Very interesting is also the asymmetry for a longitudinally polarized target which is dominated by the interference term between $\mathscr{M}_{0-,++}$ which comprises the twist- 3 effect, and the nucleon helicity-flip amplitude for $\gamma_{L}^{*} \rightarrow \pi$ transition, $\mathscr{M}_{0-, 0+}$. Results for $A_{U L}^{\sin \phi}$ are displayed and compared to the data in Fig. 4. In both the cases, $A_{U T}^{\sin \phi_{s}}$ and $A_{U L}^{\sin \phi}$, the prominent role of the twist-3 mechanism is clearly visible. Switching it off one obtains the dashed lines which are significantly at variance with experiment. In this case the transverse amplitudes are only fed by the pion-pole contribution. 
Although the main purpose of the work presented in [1] is focused on the analysis of the HERMES data one may also be interested in comparing this approach to the Jefferson Lab data on the cross sections [6]. With the GPDs $\widetilde{H}, \widetilde{E}$ and $H_{T}$ in their present form the agreement with these data is poor. It is to be stressed however that the approach advocated for in $[1,5]$ is optimized for small skewness. At larger values of it the parameterizations of the GPDs are perhaps to simple and may require improvements. It is also important to realize that the GPDs are probed by the HERMES data only for $x$ less than about 0.6. One may therefore change the GPDs at large $x$ to some extent without changing much the results for cross sections and asymmetries in the kinematical region of small skewness. For Jefferson Lab kinematics, on the other hand, such changes of the GPDs may matter.

In summary, there is strong evidence for transversity in hard exclusive electroproduction of pions. A most striking effect is seen in the target asymmetry $A_{U T}^{\sin \phi_{s}}$. The interpretation of this effect requires a large helicity non-flip amplitude $\mathscr{M}_{0-,++}$. Within the handbag approach this amplitude is generated by the helicity-flip or transversity GPDs in combination with a twist-3 pion wave function [1]. This explanation establishes an interesting connection to transversity parton distributions measured in inclusive processes. Further studies of transversity in exclusive reactions are certainly demanded. Good data on $\pi^{0}$ electroproduction would also be welcome. They would not only allow for further tests of the twist-3 mechanism but also give the opportunity to verify the model GPDs $\widetilde{H}$ and $\widetilde{E}$ as used in Ref. [1].

\section{References}

[1] S.V. Goloskokov and P. Kroll, Eur. Phys. J. C65 (2010) 137, [arXiv:0906.0460].

[2] A. V. Radyushkin, Phys. Lett. B385 (1996) 333, [hep-ph/9605431].

[3] J.C. Collins, L. Frankfurt and M. Strikman, Phys. Rev. D56 (1997) 2982, [hep-ph/9611433].

[4] J. Botts and G. Sterman, Nucl. Phys. B325 (1989) 62.

[5] S. V. Goloskokov and P. Kroll, Eur. Phys. J. C42 (2005) 281; ibid. C53 (2008) 367.

[6] H. P. Blok et al. [Jefferson Lab Collaboration], Phys. Rev. C78 (2008) 045202.

[7] A. Airapetian et al. [HERMES Collaboration], Phys. Lett. B682, 345 (2010), [arXiv:0907.2596].

[8] C. Bechler and D. Mueller, arXiv:0906.2571.

[9] A. Airapetian et al. [HERMES Collaboration], Phys. Lett. B535 (2002) 85, [hep-ex/0112022].

[10] M. Diehl, Eur. Phys. J. C19 (2001) 485, [hep-ph/0101335].

[11] P. Hoodbhoy and X. Ji, Phys. Rev. D58 (1998) 054006, [hep-ph/9801369].

[12] H. W. Huang et al., Eur. Phys. J. C33 (2004) 91, [hep-ph/0309071].

[13] H. W. Huang and P. Kroll, Eur. Phys. J. C17 (2000) 423, [hep-ph/0005318].

[14] V. M. Braun and I. E. Halperin, Z. Phys. C48 (1990) 239. [Sov. J. Nucl. Phys. 52 (1990 YAFIA,52,199-213.1990) 126].

[15] A. V. Radyushkin, Phys. Lett. B449 (1999) 81, [hep-ph/9810466].

[16] M. Anselmino et al., Phys. Rev. D75 (2007) 054032, [hep-ph/0701006].

[17] A. Airapetian et al. [HERMES Collaboration], Phys. Lett. B659 (2008) 486, [arXiv:0707.0222]. 